\title{
VALORAÇÃO CONTINGENTE DE UNIDADES DE CONSERVAÇÃO. ESTUDO DE CASO: HORTO FLORESTAL DE RONDONÓPOLIS (MT)
}

\author{
Maurício Alves Rodrigues Pugas ${ }^{1}$ \\ Heitor Lopes Ferreira ${ }^{2}$ \\ Nilton Francisco Rodrigues de Souza ${ }^{3}$ \\ Leny Kovaliski Ferreira ${ }^{4}$ \\ Alice Lopes Rodrigues Pugas ${ }^{5}$ \\ Juliana Lopes Rodrigues Pugas de Souza ${ }^{6}$
}

\section{INTRODUÇÃO}

As cidades passaram a ter um papel cada vez mais significativo no planeta, tanto em termos quantitativos como qualitativos. Em 1940, cerca de 70\% da população brasileira viviam no campo. Atualmente, estima-se que mais de $80 \%$ da população brasileira vivem em cidades. Isto acarreta uma série de problemas sociais e ambientais, que acabam tornando a "sobrevivência" nestes espaços cada vez mais difícil (BRASIL, 2003).

O homem, nos estágios tribal e rural, possuía consciência da importância da natureza para sua sobrevivência e criou mecanismos sociais para sua preservação. No entanto, o homem da cidade conhece muito pouco, a princípio, sobre o nosso ambiente natural, embora este seja uma necessidade intrínseca. Neste contexto, e de acordo com Hardt (1995), áreas verdes urbanas são áreas permeáveis, ou seja, áreas livres públicas ou não, com uma vasta cobertura vegetal predominantemente arbórea, que apresentam

1 Doutorando em Administração (UNINOVE/UFMT); Professor efetivo do curso de Administração da UFMT, Campus de Rondonópolis-MT.

2 Doutorando em Administração (UNINOVE/UFMT); Professor efetivo do curso de Administração da UFMT, Campus de Rondonópolis-MT.

3 Bacharel em Administração pela FGV e Empresário no setor alimentício.

4 Graduanda em Ciências Contábeis pela UFMT, Campus de Rondonópolis-MT.

5 Advogada e Especialista em Didática e Metodologia do Ensino Superior.

6 Bacharel em Direito 
funções potenciais capazes de proporcionar um microclima distinto no meio urbano em relação à luminosidade, temperatura e outros parâmetros associados ao bem-estar humano (funções de lazer).

Com a implantação das cidades e seu desenvolvimento, houve a diminuição das áreas verdes naturais, o que trouxe consigo o aparecimento de inúmeros problemas devidos à ausência dessas áreas. Segundo Barros (2003, p.11), "o termo impacto recreativo é utilizado para identificar os distúrbios causados pelo uso público na qualidade da experiência dos visitantes", por exemplo, lixo, fogueiras, vandalismo, entre outros. De acordo com Cole (2000), os impactos mais graves acontecem quando o número de visitantes é muito alto, quando eles apresentam comportamentos inapropriados ou ainda quando as áreas não são manejadas adequadamente.

As áreas verdes centrais, beneficiadas pelo transporte coletivo e livre acesso a freqüentadores, tornam-se, em antítese ao passado, uma fonte de lazer e recreação para as classes menos abastadas. Hoje, a cidade é o local onde reside mais da metade da população brasileira. Por isso, deve-se ter uma consideração especial com estas áreas, tratando-as como o "meio ambiente" em que o ser humano está inserido. O meio ambiente urbano deve ser organizado de tal forma que assegure uma sadia qualidade de vida (SANCHES, 2014: ROMEIRO; ANDRADE, 2009).

A manutenção, a conservação e a ampliação do número de áreas verdes urbanas constituem as principais estratégias na perspectiva da sustentabilidade ambiental e conseqüente melhoria da qualidade ambiental e de vida associada ao ambiente urbano. Por exemplo, Carneiro e outros (1993, p. 67) dizem que "o adequado gerenciamento ambiental é necessário para garantir que a degradação da natureza e a conseqüente decadência da qualidade de vida, tanto nas cidades como no campo, parem de ocorrer. E que a necessidade de se produzir seja compatível com a de se preservar o meio ambiente".

A valoração dos "bens e serviços" ambientais em unidades comparáveis aos "bens e serviços" econômicos surge como uma estratégia fundamental para sua incorporação efetiva nas decisões políticas e nas análises econômicas dos projetos de desenvolvimento (CONSTANZA, 1994; ALENCAR FILHO et al, 2013; DHOLAKIA,2010). Baseados no preço de mercado, vários métodos de 
valoração econômica têm sido desenvolvidos para quantificar os "bens e serviços" ambientais.

Os "bens e serviços" ambientais que não apresentam preços de mercado são valorados pelo Método de Valoração Contingente (MVC), tido como uma das principais ferramentas para estimar esse valor econômico. O método consiste basicamente no estabelecimento de um mercado hipotético, em que os indivíduos são questionados sobre suas preferências por um determinado "bem ou serviço" ambiental e sua Disposição a Pagar (DAP) pela sua manutenção e conservação (JANSSON, 2009; MARCANTONIO.; SCHENKEL, 2010).

Desta forma, o MVC para estimar o valor monetário que a população urbana e rural do município de Rondonópolis (MT) está disposta a pagar de forma Espontânea e Induzida, para manter e conservar o Horto Florestal do município, relacionada aos aspectos socioeconômicos, comportamentais e atitudinais, bem como, àqueles provenientes da consciência de preservação do meio ambiente, visando o seu bem-estar e de suas gerações futuras, e ainda, assegurar, efetivamente, a integração dos mesmos no planejamento e nas tomadas de decisões como subsídios para a manutenção e gestão da área natural, é de fundamental importância científica em questão.

\section{DESENHO EXPERIMENTAL}

O Horto Florestal de Rondonópolis é um bem público, está localizado no município de Rondonópolis, na região sudeste do Estado de Mato Grosso (Figura 1). Localizado a $2 \mathrm{~km}$ do centro da cidade, com 173.000 metros quadrados, além de ser um centro de preservação da natureza dentro da cidade, oferece aos esportistas uma pista de Cooper com 1.600 metros de extensão, rodeada por muito verde, e $\mathbf{2 . 4 0 0}$ metros de trilha ecológica percorridas no interior da mata, com árvores de diferentes espécies, parquinho infantil, área para ginástica aeróbica ao ar livre, galpão para criação de mudas, viveiros para produção de mudas para reflorestamento, paisagismo e fruticultura domiciliar (Figura 2).

Atualmente, compete à Prefeitura Municipal de Rondonópolis viabilizar recursos para sua manutenção, bem como, estimular pro- 
jetos de melhorias na área, visando ampliar ao máximo o bem-estar social. Não existe no órgão público municipal nenhum convênio ou parcerias para a obtenção de recursos, nem tampouco, projetos cientificamente produzidos para estímulo dessas parcerias.

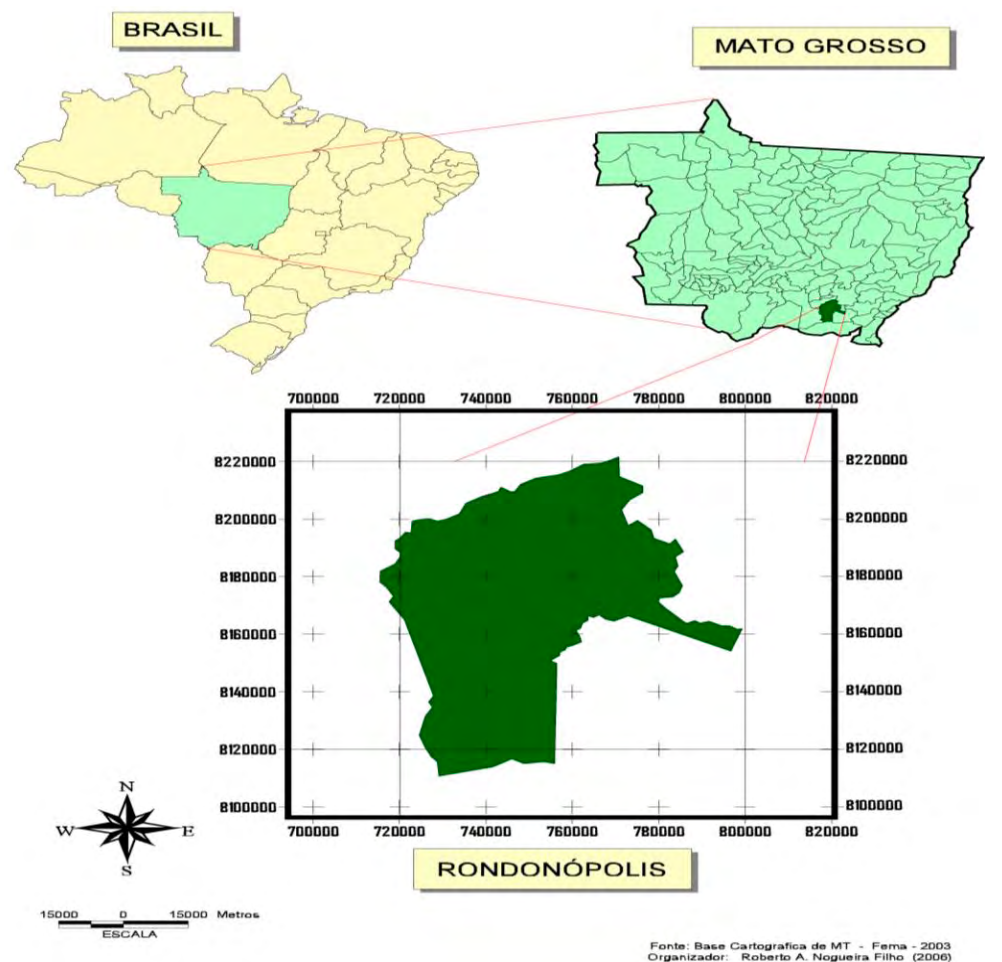

Figura 1. Mapa de Localização do Município de Rondonópolis

Fonte: MATO GROSSO. Base Cartográfica de MT, SEMA. 2017. 


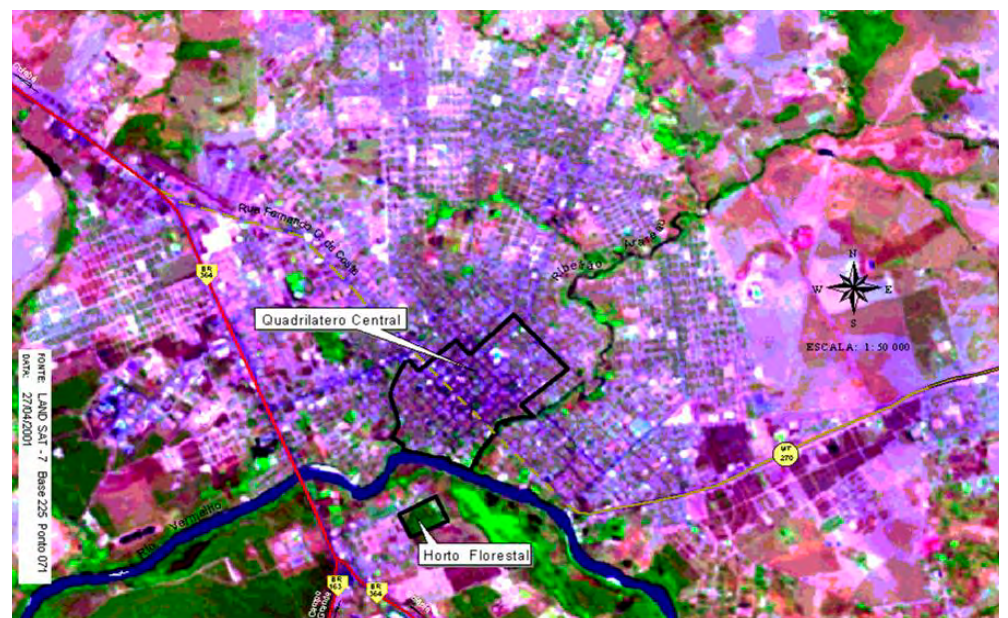

Figura 2. Localização do Horto Florestal de Rndonópolis - MT

Fonte: Arquivo da Prefeitura Municipal de Rondonópolis. 2017.

\section{METODOLOGIA}

Neste estudo a aplicação do MVC seguiu as recomendações do Painel do National Oceanic and Atmospheric Administration $(\mathrm{NOAA})^{7}(1989)$, que reconhece a validade do método de valoração contingente, desde que tomados, entre outros, os seguintes cuidados $^{8}$ : (a) adotar amostra probabilística; (b) evitar respostas nulas;

7 O NOAA foi o órgão americano designado para definir critérios e procedimentos para mensuração dos danos ambientais causados no Alaska pelo petroleiro Exxon Valdez, em 1989. Este Painel foi liderado por Robert Solow e Kenneth Arrow, dois ganhadores do Prêmio Nobel de Economia.

8 Outras condições e procedimentos recomendados para aplicação do MVC são a seguir resumidos:

- o mercado hipotético deve ser verossímil e realista;

- o veículo de pagamento utilizado e a medida de bem-estar (DAP ou DAR) não podem ser polêmicos ou invocarem oposições éticas, devendo ser neutras;

- os entrevistados devem ser abastecidos com informação suficiente a respeito do bem / serviço em questão; de preferência, os entrevistados devem ser familiarizados com o bem em questão;

- sempre que possível, a medição da DAP deve ser preferida, já que geralmente os entrevistados têm dificuldade com a noção de aceitar compensação em dinheiro em troca de mudanças no fluxo de serviços ambientais;

- uma amostra suficientemente grande deve ser adotada, para permitir um nível desejado de confiança;

- testes para vieses devem ser incluídos, e estratégias devem ser adotadas para minimizar o viés estratégico em particular;

- viés de protesto deve ser identificado;

- deve ser verificado se a amostra tem características similares às da população em 
(c) realizar entrevistas pessoais; (d) treinar os entrevistadores para serem neutros; (e) realizar pesquisas-piloto para testar o questionário; (f) oferecer informação adequada sobre o bem/serviço alvo da aplicação do método; (g) identificar uso alternativo do bem/serviço em análise; (h) administrar o tempo de pesquisa, para evitar perda de acuidade das respostas; (i) incluir outras variáveis explicativas relacionadas com o uso do recurso; (j) adotar opções que substituem a medida monetária a ser estimada, usando, por esse motivo, a DAP ao invés da DAR; ( $k$ ) identificar com clareza a alteração da disponibilidade do recurso; (I) lembrar os entrevistados da restrição orçamentária, ou seja, que a DAP resulta em menor consumo de outros bens; e $(\mathrm{m})$ apresentação dos resultados completos, com desenho da amostra, questionário, método estimativo e base de dados disponível (HANLEY; SPASH, 1993; HAIR et al, 2009).

Diante disto, os procedimentos metodológicos utilizados para a realização desta pesquisa, considerando as recomendações contidas no NOAA, envolveram as seguintes fases: (a) a definição do modelo geral aplicado aos usuários do Horto Florestal de Rondonópolis - MT, bem como, suas formas de operacionalização; (b) a delimitação e o design da pesquisa, (c) a elaboração do plano amostral; e (d) a definição da forma de coleta e do tratamento dos dados.

Como o objetivo geral deste trabalho foi o de verificar se a população urbana e rural de Rondonópolis possui a percepção de que a manutenção e a conservação do Horto Florestal aumentam o seu nível de bem-estar e, caso tenha essa percepção, aferir também se ela se dispõe a pagar por esse acréscimo em sua função de utilidade, procurou-se definir um modelo teórico que contemplasse, na literatura existente, um conjunto de variáveis que melhor descrevesse os traços gerais de características socioeconômicas; comportamentais e atitudinais; de uso e não-uso do horto, e; de consciência ecológica, com sua disposição a pagar, para justificar a manutenção e conservação do Horto Florestal de Rondonópolis-MT.

Nesta pesquisa, em um modelo de regressão múltipla, a variável dependente foi denotada por DAP (y) e as variáveis indepen-

geral, e feitos os ajustes, caso necessários; a curva de amostra deve ser estimada, e os parâmetros checados se estão de acordo com expectativas prévias. (HANLEY; SPASH, 1993; HAIR et al, 2009). 
dentes por denotações conforme a situação requerida $\left(x_{1}, x_{2}, \ldots\right.$, $x_{k}$ ). Já, para a execução da regressão linear múltipla, criou-se um modelo geral e suas respectivas formas operacionais, para posterior análise e discussão do significado de cada uma das variáveis independentes e a sua influência na disposição a pagar da população residente no município.

Considerando-se os argumentos apresentados, na definição do modelo econômico no presente estudo para a aplicação do Método de Valoração Contingente, para a obtenção da Disposição a Pagar, utilizou-se para as variáveis (dependente e independentes), as seguintes denotações e significados: Disposição a Pagar - Variável Dependente - (DAP); Localização da residência - Urbana / Rural - (ÁREA); Idade do entrevistado (IDADE); Sexo do entrevistado (SEXO); Grau de instrução (GINST); Vínculo com o chefe de família (VINC); Renda mensal pessoal (RENP); Renda mensal familiar (RENF); Número de pessoas residentes na casa (NPESS); Despesa mensal com a família - Média - (MDESF); Já ouvir falar do Horto Florestal (OFALAR); Freqüência de ida ao Horto Florestal (FREQ); Motivo preservação (MOTIV); Estado de manutenção do Horto Florestal (EMANUT); Permanência no Horto Florestal (PERM); Período de uso (PUSO); Valor de existência (VLREXIS); Valor de opção (VLROP); Valor de herança (VLRHER); Valor de uso (VLRUSO); Danos ambientais prejudicam a saúde (DANAMB); Concorda/Discorda com a manutenção (CMANUT); Interesse por temas relacionados ao Meio Ambiente (INTER); Importância pela preservação do Meio Ambiente (IMPOR); Preocupação / descaso do governo com a preservação do Meio ambiente no Brasil (GOV); e, Opinião pessoal sobre a preservação do Meio Ambiente no Brasil (OPESS).

Dessa forma, o modelo geral foi representado por:

DAP= $\mathrm{f}$ (ÁREA, IDADE, SEXO, GINST, VINC, RENP, RENF, NPESS, MDESF, OFALAR, FREQ, MOTIV, EMANUT, PERM, PUSO, VLREXIS, VLROP, VLRHER, VLRUSO, DANAMB, CMANUT, INTER, IMPOR, GOV; OPESS).

No presente estudo, utilizou-se um conjunto de variáveis que melhor identificasse as características contempladas nos entrevistados, através de quatro (4) modelos criados a partir de dois tipos de DAP's considerados: (a) DAP Espontânea (DAP Esp): que foi a manifestação dos entrevistados em pagar de imediato determinada 
quantia pela manutenção do Horto Florestal de Rondonópolis; e, (b) DAP Induzida (DAP Ind): que foi a manifestação dos entrevistados que se negaram espontaneamente a pagar de imediato qualquer quantia pela manutenção do Horto Florestal de Rondonópolis-MT, mas, que, a partir da construção de um cenário hipotético e temporal (recomendação do NOAA), no qual se consideraram que, daqui a um ano, o estado de manutenção e conservação do Horto Florestal estaria superior ao atual, com melhorias na sua infra-estrutura de lazer e recreação, incremento na sua programação cultural, educação ambiental, e outras mais, concordavam em pagar.

Os modelos operacionalizados foram os seguintes: a) Modelo I: Disposição a Pagar (Esp. e Ind.) relacionadas às características sócio-econômicas dos entrevistados; b) Modelo II: Disposição a Pagar (Esp. e Ind.) relacionadas ao comportamento e atitude dos entrevistados; c) Modelo III: Disposição a Pagar (Esp. e Ind.) relacionados aos valores de uso e não-uso; e, d) Modelo IV: Disposição a Pagar (Esp. e Ind.) relacionados à consciência ecológica.

Para cada variável independente nos modelos propostos, foram utilizadas variáveis dummy nas análises e nos modelos de regressão. Tendo em vista alcançar os objetivos propostos neste trabalho, foi realizado um delineamento do tipo levantamento-survey (SELLINTZ, WRIGHTASMAN e COOK, 1987 citados por MALHOTRA, 2011).

Desta forma, projetou-se uma pesquisa dividida nas 02 (duas) etapas: Exploratória-Qualitativa e Descritiva-Quantitativa. A etapa exploratória-qualitativa foi desenvolvida buscando atingir os seguintes objetivos principais: a) Formulação do problema de pesquisa, objetivos gerais e secundários, de maneira clara e mensurável; b) Elaboração de hipóteses a serem testadas na fase quantitativa; e, c) Desenvolvimento do modelo geral de regressão, bem como, suas formas de operacionalização a serem testadas também na fase quantitativa. E, finalmente, a etapa descritiva-quantitativa que compreendeu especificamente os quatro modelos de DAP's (Espontânea e Induzida) relacionadas às características específicas constantes do questionário.

O método para a coleta de dados aplicados nesta pesquisa foi o survey por ser um método para se obter informação baseado no questionamento aos respondentes, geralmente de forma estrutu- 
rada (MALHOTRA, 2011). Em função das características da presente pesquisa (mensuração das opiniões dos respondentes sobre seus relacionamentos com membros de um canal de distribuição), o método survey foi o método mais indicado, com a aplicação do questionário por meio presencial, face às características atribuídas ao presente estudo no sentido de contemplar as recomendações do NOAA.

No critério de amostragem, tendo como base a população urbana e rural deste estudo um total de 60.339 domicílios no município de Rondonópolis (MT), de acordo com o censo nacional realizado em 2010 pelo IBGE e estimado para 2017 (IBGE, 2010), o procedimento amostral utilizado foi o método probabilístico aleatório de sorteio no qual todas as residências do município (área rural e urbana) tinham a mesma chance de participar da pesquisa, respeitada a condição do percentual de domicílios nas áreas rurais (aproximadamente 10\%) e urbanas (aproximadamente 90\%) em relação ao total de domicílios.

Justificou-se eleger o número de domicílios como população do estudo ao invés do número de pessoas residentes do município, pelo fato de se querer obter a DAP de uma amostra significativa dos residentes do município em relação à localização das suas residências do Horto Florestal. Ainda, a população vislumbrada por esta pesquisa traz como característica o fato do respondente ter pelo menos 20 anos de idade. Estes critérios visaram assegurar a garantia da valoração da pesquisa, a qual possuiu caráter, sobretudo, subjetivo. Com estes filtros aplicados - idade e residência - garantiu-se que a parcela da amostra entendesse claramente o significado do estudo, o que permitiu ao entrevistado maior censo crítico.

Sendo assim, para a determinação do tamanho da amostra, adotou-se como referenciais estatísticos um intervalo de confiança de 95\%, proporção estimada (p) 50\% e margem de erro de + ou $-5 \%$ sobre a quantidade total de domicílios registrados no censo do IBGE (60.339). De acordo com Malhotra (2011), considerando o valor 1,96 para " $Z$ " (número de erros padrão que um ponto dista da média) e nos referenciais estatísticos estabelecidos acima, o tamanho da amostra pode ser determinado pela seguinte fórmula: 
$\mathrm{n}_{0}=Z^{2} \cdot(\mathrm{p} .(1-\mathrm{p})) / \mathrm{p}^{2}$

onde:

$\mathrm{n}_{0}=$ primeira aproximação do tamanho da amostra

Z= número de erros padrão

$\mathrm{p}=$ proporção estimada

Substituindo os valores correspondentes tem-se:

$\mathrm{n}_{0}=(1,96)^{2} \cdot\left(0,5 \cdot(1-0,5) /(0,5)^{2}\right.$

$\mathrm{n}_{0}=384,16$ domicílios

Para o efetivo cálculo do tamanho da amostra de população finita (n), considerando " $\mathrm{n}_{0^{\prime}}, \mathrm{em}$ (1), o valor do tamanho da amostra pode ser calculado através da fórmula:

$n=\left(n_{0} \cdot N\right) /\left(n_{0}+N-1\right)$

onde:

$\mathrm{n}=$ tamanho da amostra de população finita

$\mathrm{N}=$ tamanho da população

então,

$$
\begin{aligned}
& \mathrm{n}=(384,16.60 .339) /(384,16+60.339-1) \\
& \mathrm{n}=381,7359303424 \\
& \mathrm{n}=\mathbf{3 8 2} \text { domicílios. }
\end{aligned}
$$

Desta forma, o tamanho da amostra totalizou em 382 (trezentos e oitenta e dois) domicílios, sendo que, 39 (trinta e nove) domicílios em áreas rurais e 343 (trezentos e quarenta e dois) domicílios na área urbana. Para a realização do sorteio dos domicílios que foram entrevistados nas áreas rurais, foram distribuídos aleatoriamente, por sorteio, os 39 questionários entre todas as zonas rurais do município. Já para o sorteio dos domicílios da zona urbana, utilizou-se o número de cadastro do imóvel junto à secretaria tributária da Prefeitura Municipal de Rondonópolis-MT.

As entrevistas com informantes-chave foram utilizadas para melhorar a qualidade do questionário pré-elaborado. Um teste-piloto foi realizado com especialistas, mestres e doutores ligados às Instituições, entidades e ONG's residentes no município de Rondonópolis. Estes procedimentos permitiram: (a) a verificação do método de seleção e identificação dos domicílios, (b) o teste dos instrumentos de coleta (questões, formato do questionário, material de apoio visual, etc), (c) a verificação do tempo médio gasto na aplicação de cada questionário; (d) principais problemas 
e dificuldades observadas pelo entrevistador e pelos entrevistados; e (e) a testagem dos três tipos de técnicas para incitar a DAP dos entrevistados: questões abertas, jogos de lances e uso do cartão de pagamento.

As questões foram ordenadas de acordo com as recomendações do Painel do NOAA, incorporando uma série de questões, devidamente elaboradas e pré-testadas, com o intuito de não irritar ou ofender as pessoas pesquisadas. A forma de se obter a resposta da população urbana e rural do município foi a open-ended, por meio da aplicação de questões abertas, apresentando a seguinte questão: "Quanto você está disposto(a) a pagar pela manutenção e conservação do Horto Florestal de Rondonópolis?", procurando-se captar a máxima disposição a pagar dos entrevistados.

De acordo com Maia (2002, p. 35), “Apesar das limitações, o formato aberto pode ser recomendado em algumas situações definidas, onde predominem os valores de uso do recurso, ou a existência de permissões seja viável (como tarifas de visitação num parque) ". Neste caso, o painel NOAA recomenda o uso de um novo cenário a fim de se captar uma nova DAP - chamada de DAP INDUZIDA, o que foi prontamente realizado nesta pesquisa.

O tratamento dos dados foi baseado em modelos econométricos de regressão, como forma a contemplar os modelos teóricos propostos. Os modelos foram submetidos à verificação da normalidade dos dados utilizando o Teste-Kolmogorov Smirnov (PRADO, 2004; HAIR et al, 2005). A base de dados foi submetida a uma análise estatística de normalidade uni e multivariada. Adicionalmente, ponderações de linearidade e multicolinearidade (HAIR et al, 1998; 2005) também foram executadas.

A multicolinearidade refere-se à correlação entre três ou mais variáveis (HAIR et al, 1998; 2005). Isto significa que não podem existir correlações altas entre si, diga-se maior de $r= \pm 0,90$. Quanto ao tratamento de dados para o teste dos modelos, empregou-se a análise de regressão linear. As variáveis foram inseridas no modelo todas de uma vez (método enter). Os indicadores de ajustamento dos modelos considerados foram: beta padronizado, $\mathrm{R}^{2}$ do modelo, p-valor (significância).

Para o cálculo da DAPTotal (DAPT) tanto a espontânea (Esp) como a induzida (Ind), baseado nos dados obtidos na pesquisa 
tem-se o levantamento da disposição a pagar dos entrevistados, distribuído em intervalos de valores associados a uma disposição a pagar média, calcula-se a disposição a pagar total (DAPT), através da multiplicação da disposição a pagar média por uma estimativa da proporção da população da cidade relativa aos bairros existentes analisados na amostra, expressa na seguinte fórmula:.

$$
\begin{aligned}
& \text { DAPT = } \sum \text { DAPMi }[\mathrm{ni} / \mathrm{N}] \text {.(população da cidade) } \\
& \text { Onde: } \\
& \text { DAPM = disposição a pagar média } \\
& \text { ni = número de entrevistados dispostos a pagar } \\
& \mathrm{N}=\text { número total de pessoas entrevistadas } \\
& \mathrm{i}=\text { um dos intervalos separados } \\
& \mathrm{n}=\text { número de intervalos separados. }
\end{aligned}
$$

FONTE: Motta (1998, p.27).

Na avaliação das estimativas de DAP ou DAC deve-se considerar sua aceitabilidade em alguns aspectos relativos à sua confiabilidade, à sua validade e aos seus vieses.

\section{RESULTADOS E DISCUSSÃO}

O questionário utilizado na coleta de dados compreendeu quatro momentos, a saber: (a) Inicialmente, após a apresentação do pesquisador, foi realizada uma filtragem do entrevistado, com o propósito de identificá-lo quanto ao enquadramento do perfil do participante - residência em Rondonópolis e idade; (b) Logo após, questões sobre as características sócio-econômicas dos entrevistados - sexo, escolaridade, profissão, renda, entre outros; (c) Como um terceiro passo, foram aplicadas questões para averiguar as opiniões e atitudes dos entrevistados em relação ao Horto Florestal, mediante uma descrição geral do "bem" a ser valorado e estimulando os entrevistados à DAP para a manutenção e conservação do Horto Florestal; e, (d) Como um último momento, questões com informações sobre a consciência ecológica, com o propósito de identificar o padrão de comportamento do entrevistado como fator de influência direta no resultado da disposição a pagar. 
É importante ressaltar que, neste estudo, a população entrevistada mostrou-se bastante receptiva e interessada em colaborar com a pesquisa. A seqüência das questões, a familiaridade com a área em estudo, e a interface do meio ambiente com o bem-estar dos indivíduos, associadas à diversidade de "bens e serviços" que as áreas naturais podem fornecer, direta ou indiretamente, à sociedade, foram fatores determinantes na interação dos entrevistados com o tipo de pesquisa desenvolvido.

Inicialmente quanto à caracterização sócio-econômica dos entrevistados, constatou-se que a maior predominância estava situada na faixa etária de 20 a 25 anos, representando $33,60 \%$ dos entrevistados, seguida pela faixa etária de 26 a 35 anos (32,81\%), totalizando $66,41 \%$, representando a opinião de mais da metade dos entrevistados. Quando realizada a estatística descritiva da faixa etária dos entrevistados, constatou-se uma idade média de 32 anos, variando de 20 a 76 anos. A variação entre máximo e mínimo é importante ao trabalho por tentar refletir diferentes opiniões dos respondentes, não viesando a amostra para apenas uma dada faixa etária.

Quanto ao grau de escolaridade dos entrevistados, constatou-se que a maior parcela foi representada por pessoas que informaram ter cursado até o $2^{\circ}$ Grau Completo (32,02\%), seguido dos que declararam possuir curso superior incompleto $(28,08 \%)$, representando mais da metade $(60,10 \%)$ dos entrevistados. Estudos de especialização, mestrado e doutorado correspondem a apenas $1,31 \%$. Isto permitiu ao pesquisador concluir que a população entrevistada possui um elevado índice de escolaridade e é possível que sejam portadores de conhecimentos sobre as questões ambientais.

Com relação à renda individual, os resultados mostram que $39,90 \%$ dos entrevistados declararam possuir uma renda individual mensal de até $R \$ 500,00$, seguidos de $22,05 \%$ entre $R \$ 501,00$ a $\mathrm{R} \$ 1.000,00$ e $20,47 \%$ entre $\mathrm{R} \$ 1.001,00$ a $\mathrm{R} \$ 2.000,00$. As faixas de rendimentos acima representam cumulativamente $82,42 \%$ dos que ganham entre $R \$ 500,00$ e $R \$ 2.000,00$. Quando analisada a renda familiar, verifica-se que a faixa de renda de $\mathrm{R} \$ 1.001,00$ a $\mathrm{R} \$$ $2.000,00(38,58 \%)$ predomina sobre as demais, seguida da faixa de $\mathrm{R} \$ 2.001,00$ a $\mathrm{R} \$ 3.000,00(22,31 \%)$, culminando com a faixa de $\mathrm{R} \$ 501,00$ a $\mathrm{R} \$ 1.000,00$ (18,11\%), sendo as mais representativas. 
Observa-se ainda que 10,76\% possuem renda familiar superior a $\mathrm{R} \$ 4.000,00$. Analisando-se os gastos mensais com o número de pessoas residentes, constata-se uma média de 3,6 pessoas por família (Tabela 1).

\begin{tabular}{ccc}
\hline Variáveis & Média & Desvio padrão \\
\hline Número de pessoas residentes & 3,60 & 1,01 \\
\hline Gastos com alimentação (reais) & 373,13 & 275,39 \\
\hline Gastos com moradia (reais) & 68,15 & 193,58 \\
\hline Gastos com lazer (reais) & 159,55 & 142,94 \\
\hline Gastos com transporte (reais) & 205,17 & 144,16 \\
\hline Gastos com educação (reais) & 257,68 & 530,95 \\
\hline Gastos com água (reais) & 36,39 & 22,92 \\
\hline Gastos com luz (reais) & 160,85 & 97,70 \\
\hline Gastos com saúde (reais) & 122,84 & 137,15 \\
\hline Média (gastos e desvio padrão) & 172,97 & 193,10 \\
\hline
\end{tabular}

Tabela 1 - Estatísticas descritivas do $\mathrm{n}$ o de pessoas e os gastos mensais Fonte: Construção dos autores.

Quanto ao comportamento e atitude dos entrevistados em relação ao Horto, constou-se que em relação à manutenção e conservação do Horto Florestal, 88,71\% já ouviram falar do Horto Florestal e visitaram o Horto, e apenas $11,29 \%$ já ouviram falar, mas nunca o visitaram. Com relação à freqüência, $21,52 \%$ vão uma vez por ano ao Horto, seguidos de $14,70 \%$ dos que freqüentam apenas uma vez ao semestre, 14,44\%, apenas uma vez por mês, e 14,17\% apenas uma vez a cada dois meses. Do total acima, somando-se os que visitaram menos vezes (17,32\%), se constata que a maioria $(82,15 \%)$ já visitou o Horto Florestal e conhece suas necessidades.

Quase a totalidade da população entrevistada (97,38\%) possui elevado nível de conhecimento sobre a preservação e manutenção do Horto Florestal de Rondonópolis. Ainda quanto à freqüência ao Horto, pelo fato da maioria da população entrevistada demonstrar consciência da necessidade de se preservar e manter conservado o Horto Florestal, e os questionários terem sido aplicados nos 
domicílios, reflete que a população residente, e não aqueles contumazes freqüentadores diários estão realmente dispostos a pagar pela manutenção e conservação do Horto Florestal.

Considerando o estado de conservação e manutenção do Horto Florestal de Rondonópolis, 92,71\% consideram seu estado de conservação e manutenção de bom a muito bom, representando a maioria dos entrevistados. Apesar do estado de conservação e manutenção ter sido considerado de bom a muito bom, ainda $87,14 \%$ dos entrevistados consideram que o Horto Florestal de Rondonópolis necessita de melhorias; e entre as melhorias citadas, o item "uma maior divulgação" recebeu maior número de votos, seguida de manutenção e da realização de eventos culturais.

Como já discutido na revisão teórica, há uma correlação entre a existência de áreas verdes urbanas e o nível de bem-estar da população residente. Esta também é uma preocupação dos entrevistados nesta pesquisa, pois $91,34 \%$ concordam que a manutenção e conservação do Horto Florestal têm influência no bem-estar das pessoas. Considerando-se que $73,75 \%$ manifestaram satisfação quanto ao seu bem-estar no Horto Florestal de Rondonópolis, ainda uma parcela significativa $(26,25 \%)$ acha que faltam incentivos de melhorias na área ambiental preservada.

$\mathrm{Na}$ análise da disposição a pagar tal como mencionado anteriormente, a disposição a pagar dos entrevistados, com o intuito de possibilitar a manutenção e conservação do Horto Florestal, foi obtida em dois momentos: (a) por meio da DAP espontânea e, (b) por meio da DAP induzida. Os resultados mostraram que apenas $30,71 \%$ manifestaram interesse a pagar espontaneamente para a manutenção e conservação do Horto Florestal de Rondonópolis. As respostas apresentaram um percentual de $14,44 \%$ para o valor de $\mathrm{R} \$$ 5,00 por ano, representando a intenção de quase a metade dos que se interessa em pagar, e os demais percentuais, valores compreendidos entre $\mathrm{R} \$ 1,00$ até $\mathrm{R} \$ 200,00$ anuais.

Do total de 382 entrevistados, 69,29\% manifestaram que não estariam dispostos a pagar para a manutenção e conservação do Horto Florestal. Observou-se que a faixa etária entre 20 e 65 anos, apresentou o maior percentual dos votos de protesto, e acima de 65 anos, os maiores percentuais nas faixas dos valores ( $R \$ 1,00$ a $\mathrm{R} \$ 20,00)$ para a disposição a pagar pela manutenção e conserva- 
ção do Horto Florestal. Tal comportamento refletiu que, pessoas amadurecidas são mais conscientes da necessidade de preservar o meio ambiente para si e para suas gerações futuras.

Desse percentual $(69,29 \%)$, diversas foram às razões apresentadas para manifestar sua indisposição a pagar. Identificou-se os principais motivos: $25 \%$ transferiram a responsabilidade para a Prefeitura Municipal, 13\% disseram que é dever do Governo, $5 \%$ alegaram que já pagam imposto suficiente, $6 \%$ caracterizaram que não é dever da população, Apenas $2 \%$ alegaram que não têm condições financeiras (o valor da manifestação corresponde a $\mathrm{R} \$$ $0,00)$, e, finalmente, $18 \%$ alegaram motivos diversos. Diante do exposto, concluiu-se que uma parcela muito pequena demonstrou falta de condições financeiras, caracterizando, então, que a maioria dos que se indispuseram a pagar, manifestou votos de protesto.

Neste caso, em atenção às recomendações do NOAA, em que os vieses de protestos devem ser identificados, partiu-se, então, para a obtenção da DAP induzida. Para o total de 69,29\% dos que não manifestaram disposição a pagar espontânea pela conservação e manutenção do Horto Florestal de Rondonópolis, elaborou-se um cenário alternativo e hipotético para um período $t+1$, ou seja, um novo cenário foi apresentado ao respondente, considerando que 01 (um) ano depois, estando com melhorias na sua infra-estrutura de lazer e recreação, incremento na programação cultural, educação ambiental, entre outras, perguntou-se novamente sobre a disposição a pagar anualmente na forma de imposto, em reais, para manter as funções do Horto florestal. A partir da DAP induzida, 264 pessoas entrevistadas $(69,29 \%)$ sinalizaram valores que variaram de $R \$ 2,00$ a $R \$ 50,00$, sendo $R \$ 5,00$ e $R \$ 8,00$ os mais expressivos.

Agregando-se os resultados obtidos da DAP espontânea e da DAP induzida, verifica-se que as respostas para a DAP induzida apresentaram $R \$ 2,00$ anuais para valor mínimo e $\mathrm{R} \$ 50,00$ anuais como valor máximo. Considerando-se os valores apresentados, observa-se que a média do valor declarado espontaneamente foi de $R \$ 4,68$, com desvio padrão de 17,02 , e a média declarada induzida alterou a DAP para $\mathrm{R} \$ 9,85$ per capita/ano, com um desvio padrão de 9,49 (Tabela 2). 


\begin{tabular}{cccccc}
\hline & N & $\begin{array}{c}\text { Mínimo } \\
\text { (R\$) }\end{array}$ & $\begin{array}{c}\text { Máximo } \\
\text { (R\$) }\end{array}$ & $\begin{array}{c}\text { Média } \\
\text { (R\$) }\end{array}$ & Desvio Padrão \\
\hline DAP Espontânea & 382 & 0,00 & 200,00 & 4,6824 & 17,02201 \\
DAP Induzida & 210 & 2,00 & 50,00 & 9,8500 & 9,49000 \\
\hline
\end{tabular}

Tabela 2 - Estatística Descritiva

Fonte: Construção dos autores.

Quando realizada a regressão linear da DAP e as variáveis binárias socioeconômicas, constatou-se que apenas a renda oferece consistência na variação da disposição a pagar, visto o valor do grau de significância se enquadrar menor que 0,05 (Tabela 3).

\begin{tabular}{c|c|c|c|c|c}
\hline \multirow{2}{*}{ Modelo } & \multicolumn{2}{|c|}{$\begin{array}{c}\text { Coeficiente } \\
\text { Não padronizado }\end{array}$} & $\begin{array}{c}\text { Coeficiente } \\
\text { Padronizado }\end{array}$ & \multirow{2}{*}{ T } & Signific. \\
\cline { 2 - 4 } & B & Erro Padrão & Beta & T & ,006 \\
\hline Constante & 16,590 & 6,054 & & 2,741 &, 480 \\
\hline Área & $-2,041$ & 2,888 &, 036 &,- 707 &, 419 \\
\hline Idade &,- 065 &, 080 &,- 044 &,- 809 &, 273 \\
\hline Vínculo & $-2,146$ & 1,955 &, 058 & $-1,098$ &, 001 \\
\hline Renda & $-6,361$ & 1,885 &,- 185 & $-3,374$ &, 335 \\
\hline N. Pessoas &,- 885 &, 916 &,- 053 &,- 966 &, \\
\hline
\end{tabular}

Tabela 3 - Regressão Linear (Coeficientesaa)

a.Variável Dependente $=$ DA

Fonte: Construção dos autores.

Observou-se ainda, quando realizada a regressão linear da DAP e a Renda, considerando-se o valor de 1 (um) para a renda até $R \$ 2.000,00$ e 0 (zero) para renda acima de $R \$ 2.000,00$, que a Disposição a Pagar de quem ganha acima de $\mathrm{R} \$ 2.000,00$ é menor de quem ganha até $\mathrm{R} \$ 2.000,00$.

Quando perguntado qual o melhor motivo para o entrevistado ter a disposição a pagar para preservar o Horto Florestal, 61\% manifestaram interesse pela conservação por proporcionar aos familiares passeios ao ar livre (caminhadas) e pelo lazer no campo, como bem-estar coletivo (valor de uso), 22\% para geração futura e medicina (valor de herança), 10\% para a existência de animais e plantas (valor de existência) e finalmente, $7 \%$ para vê-lo no futuro (valor de opção).

$\mathrm{Na}$ análise da consciência ecológica, os entrevistados apontaram uma série de fatores indicativos da problemática de risco ambiental que tem contribuído para o processo de degradação das 
áreas do Horto Florestal de Rondonópolis. Inicialmente, a maioria $(99,21 \%)$ concordou que queimadas, poluições dos rios e desflorestamentos causam danos ambientais, prejudicando a saúde. Dentre os danos ambientais citados anteriormente, $51,44 \%$ considera a poluição dos rios um dos problemas ambientais mais preocupantes, seguida de desflorestamentos $(30,45 \%)$ e queimadas $(16,80 \%)$, refletindo a preocupação de $98,69 \%$ da população entrevistada.

Quando solicitado sua opinião sobre a manutenção e conservação do meio ambiente, trazendo melhorias para as condições de vida da sociedade, 98,95\% concordaram, restando apenas $1.04 \%$ indiferentes à situação. Quanto ao interesse por temas relacionados ao meio ambiente, $72,11 \%$ demonstraram ser o meio ambiente interessante e muito interessante, restando apenas $27,89 \%$ sem considerações e sem interesses pelo meio ambiente ou ecologia. Quase a totalidade $(97,37 \%)$ dos entrevistados declarou ser a preservação do meio ambiente importante e muito importante no país.

Com relação à sua preocupação quanto ao governo brasileiro no que se refere à preservação do meio ambiente no país, apenas $40,68 \%$ declararam preocupado e muito preocupado, ao passo que os demais 59,32\% indiferença e despreocupação. Isto reflete que a maioria concorda com o fato de que a população tem que se mobilizar a fim de gerar recursos para preservar o meio ambiente.

Finalizando, concluiu-se a pesquisa atribuindo aos entrevistados sua manifestação quanto à responsabilidade pela preservação do meio ambiente no Brasil em dois momentos, e contatou-se que $59,58 \%$ e $65,09 \%$, respectivamente, atribuem ao governo (federal, estadual e municipal) a responsabilidade pela preservação e os demais, $(40,42 \%)$ e $(34,91 \%)$, das ONG's e população. Isto evidencia o viés de protesto identificado na obtenção da DAP dos entrevistados.

Definidos os modelos econômicos para a aplicação do Método de Valoração Contingente, após a seleção do conjunto de variáveis independentes, o próximo procedimento foi da estimação dos modelos. O propósito foi obter a forma estrutural que apresentasse 0 melhor comportamento para a relação das variáveis independentes com a variável dependente, em cada situação criada, ou seja, para cada tipo de variável dependente (espontânea e induzida). E, desta forma, obter os melhores resultados sob a perspectiva estatística 
e econométrica, através da discussão dos resultados, por meio da análise de regressão e da lógica econômica.

Antes das análises dos modelos propostos, foram efetuados os seguintes testes na base de dados: (a) Normalidade; (b) Linearidade; e, (c) Multicolineariedade (HAIR et al, 2009), com o intuito de verificar a confiabilidade do mesmo e melhorar o desempenho dos modelos testados. Segue abaixo uma descrição resumida de cada um destes testes:

a. Normalidade: Vale salientar que todas as variáveis foram significativas no que tange ao teste Kolmogorov Smirnov $(p<, 01)$, indicando que os dados são não-normais (BISQUERRA; SARRIERA; MARTÍNEZ, 2004; TABACHNICK e FIDELL,2007).

b. Linearidade: A matriz de correlação das variáveis é mostrada na tabela 4 a seguir. Devido ao fato de que algumas variáveis são dummy e outras são métricas, apresenta-se nesta tabela apenas o valor de correlação bivariada de Pearson das variáveis métricas. Notam-se alguns valores negativos, outros positivos e outros sem significância. Por exemplo, quanto mais novo (idade), menor é a disposição de pagamento de manutenção do Horto, ou seja, os mais novos tendem a transferir a responsabilidade pela preservação e conservação a terceiros - ONG'S, governo federal, estadual e municipal. (TABELA 4).

c. Multicolinearidade: A multicolinearidade refere-se à correlação entre três ou mais variáveis independentes (HAIR et al, 1998; 2009). Isto significa que não podem existir correlações altas entre si, diga-se maior de $r= \pm 0,90$. Os valores de colinearidade foram verificados dentro da tabela por meio da correlação de Pearson (Tabela 4). Os resultados não apresentaram colinearidade das variáveis.

\begin{tabular}{llllllllllll}
\hline 1 & 2 & 3 & 4 & 5 & 6 & 7 & 8 & 9 & 10 & 11 & 12 \\
\hline
\end{tabular}

1. IDADE

2. NPESS,$- 154^{* *}$

3. Moradia $\quad-, 075 \quad-, 035$

4.Alimentação ,219**, 329** ,078

5. Lazer $\quad-372 * *$

6.Transporte - $\quad$ 099 349, 351**, 063**

7. Educação - $\quad$-183* ,310**

Continua... 


\begin{tabular}{|c|c|c|c|c|c|c|c|c|c|c|}
\hline 8. Agua &,$- 152 *$ &, $303 * *$ &,- 027 &, $264 * *$ & , 240** & $230 * *$ & $208^{* *}$ & & & \\
\hline 9. Luz &,- 029 &, $310 * *$ &,- 028 &, $406 * *$ & , 408** &, $451 * *$ & $297 * *$ & , 521** & & \\
\hline 10. Saude &, $302 * *$ & 104 &,- 095 & ,045 &, $228 * *$ & $340 * *$ & ,072 &, $233 * *$ & 125 & \\
\hline 11.DAP_IND &,- 036 & ,041 & 104 &,- 039 &,- 029 & ,024 & ,018 & -122 & ,005 & ,069 \\
\hline 12. DAP_ESP &,- 088 & ,027 & ,199* &, $344 * *$ &, $308 * *$ & 183 &, $442^{* *}$ & , 222* &, $227 *$ &, $028 \quad$ (a) \\
\hline
\end{tabular}

Tabela 4 - Matriz de correlação de Pearson

** Correlação significativa no nível de 0,01 (2 tailed).

* Correlação significativa no nível de 0,05 (2-tailed).

a. Não pode ser calculado

Fonte: Construção dos autores.

Após a verificação da linearidade e multicolinearidade, foram testados todos os modelos de regressão sugeridos por este estudo. O modelo I tinha as variáveis independentes iguais, contudo, a única variável que apresentou variação no modelo, foi a variável endógena. Assim, quando a variável dependente foi DAP Esp., os resultados apresentaram a GINST como preditora significativa no modelo, com valor do $\mathrm{R}^{2}=0,127$ (ajustado). Como a GINST deu positiva, quanto mais alto for o nível de instrução do respondente, maior será a intenção de manter o Horto. No modelo com DAP induzida, nenhum resultado foi significativo em termos de predição da variável dependente (Tabela 5).

\begin{tabular}{c|c|c}
\hline VARIÁVEIS & \multicolumn{2}{|c}{ MODELO I } \\
\hline CONSTANTE & DAP ESPONTÂNEA & DAP INDUZIDA \\
\hline ÁREA & $-49,022$ & 9,83 \\
\hline IDADE & 0,083 & 0,024 \\
\hline SEXO & 0,07 & $-0,029$ \\
\hline GINST & $-0,228$ & $-0,029$ \\
\hline VINC & $0,240 *$ & 0,013 \\
\hline RENP & 0,24 & $-0,119$ \\
\hline RENF & 0,204 & $-0,075$ \\
\hline NPESS & 0,091 & $-0,017$ \\
\hline MDESF & $-0,022$ & 0,127 \\
\hline
\end{tabular}

Tabela 5 - Resultados das DAP's do Modelo I

Nota: ${ }^{*} p<0,05 ;{ }^{* *} p<0,01 ;{ }^{* * *} p<0,001$; valores Beta padronizados

Fonte: Construção dos autores. 
O modelo I apresentou como resultado principal que o GINST (grau de instrução) foi um preditor significativo da DAP Esp. quando diz respeito à disposição a pagar.

O modelo II, a variável OFALAR foi excluída automaticamente do modelo de regressão pelo software, pois ela não teve variância. Isto significa que todos seus resultados, quando solicitada uma análise descritiva, foram 1 (dentro de uma resposta apenas). Ou seja, todos os respondentes preencheram apenas um valor na variável. Quando a variável dependente foi DAP Espontânea, os resultados apresentaram EMANUT como a variável exógena negativamente significativa no modelo ( $p<0,055)$. O valor do $R^{2}$ foi 0,002 (ajustado), ou seja, bem baixo. O modelo da DAP Induzida mostrou-se com nenhum valor significativo no que tange à associação das variáveis (Tabela 6).

\begin{tabular}{c|c|c}
\hline VARIÁVEIS & \multicolumn{2}{|c}{ MODELO II } \\
\hline CONSTANTE & DAP ESPONTÂNEA & DAP INDUZIDA \\
\hline OFALAR & 17,704 & 5,372 \\
\hline FREQ & - & - \\
\hline MOTIV &,- 021 &, 09 \\
\hline EMANUT &,- 017 &,- 007 \\
\hline PERM &,$- 102^{*}$ &,- 023 \\
\hline PUSO &,- 05 &,- 045 \\
\hline
\end{tabular}

Tabela 6 - Resultados das DAP's do Modelo II

Nota: ${ }^{*} p<0,05 ;{ }^{* *} p<0,01 ; * * * p<0,001 ;$ valores Beta padronizados; variável OFALAR não foi introduzida devido a não variância.

Fonte: Construção dos autores.

Neste modelo, o resultado uniforme em que a manutenção foi um preditor negativamente significativo da DAP. Negativo significa a visão de que o Horto está com um estado de "muito ruim a regular", ao invés do positivo de "1", para estado de "bom a muito bom".

Com relação ao modelo III, a variável dependente inicialmente foi DAP Esp. Os resultados apresentaram EVLREXIS, VLROP e VLRUSO como significativas na predição da endógena. $O$ valor do $R^{2}$ $=0,16$ (ajustado). Esse valor de $R^{2}$ foi um dos mais altos achados até o momento. Os valores esperados, conforme mencionado 
anteriormente, eram positivos, e de fato, isto ocorreu, sendo que as três variáveis foram positivas em predizer DAP Esp (Tabela 7).

\begin{tabular}{c|c|c}
\hline VARIÁVEIS & \multicolumn{2}{|c}{ MODELO III } \\
\hline & DAP ESPONTÂNEA & DAP INDUZIDA \\
\hline CONSTANTE & 4,269 & 10,047 \\
\hline VLREXIS &, $376^{* * *}$ &,- 031 \\
\hline VLROP &, $028^{* * *}$ &, 090 \\
\hline VLRHER &, 194 &,- 039 \\
\hline VLRUSO &, $13^{* *}$ &,- 046 \\
\hline
\end{tabular}

Tabela 7 - Resultados das DAP's do Modelo III Nota: ${ }^{*} p<0,05 ;{ }^{* *} p<0,01 ; * * *<<0,001$; valores Beta padronizados; Fonte: Construção dos autores.

Os resultados apresentaram VLREXIS (em função de as pessoas serem individualistas, e atribuírem ao seu bem-estar condição única, o sinal esperado é positivo) e VLROP (O sinal esperado foi positivo, porque as pessoas têm no meio ambiente uma alternativa para o aumento de seu bem-estar, e o Horto Florestal poderá Ihes oferecer esta opção no futuro) como significativas no modelo, sendo apenas a primeira positiva. Novamente, nenhuma variável mostrou-se significativa em predizer a DAP induzida, gerando, com isso, dois modelos de regressão com o pressuposto da DAP induzida como dependente, mas nenhuma deu significância.

O último modelo foi o IV, novamente com as duas DAP's como dependentes. Na variável DAP Esp. apenas uma variável foi significativa, ou seja, apenas OPESS prediz negativa e significativamente. $O$ valor do $R^{2}=0,02$ (ajustado), sendo muito baixo. No modelo com DAP induzida nenhuma variável mostrou-se significativa na predição (Tabela 8).

\begin{tabular}{c|c|c}
\hline VARIÁVEIS & \multicolumn{2}{|c}{ MODELO IV } \\
\hline & DAP ESPONTÂNEA & DAP INDUZIDA \\
\hline CONSTANTE & 24,267 & 6,81 \\
\hline DANAMB &,- 079 &,- 034 \\
\hline CMANUT &, 025 &, 009 \\
\hline INTER &,- 023 &, 043 \\
\hline
\end{tabular}

Continua... 


\begin{tabular}{c|c|c}
\hline IMPOR &, 014 &, 053 \\
\hline GOV &, 013 &, 093 \\
\hline OPESS &,$- 105^{*}$ &,- 022 \\
\hline
\end{tabular}

Tabela 8 - Resultados das DAP's do Modelo IV Nota: ${ }^{*} p<0,05$; ${ }^{*} p<0,01 ;{ }^{* *} p<0,001$; valores Beta padronizados; Fonte: Construção dos autores.

Neste modelo, variáveis que foram significativas e similares dentro das duas DAP's, como homogêneas, não apareceram. Assim, na variável DAP Esp., apenas uma variável foi significativa, ou seja, apenas OPESS predisse negativa e significativamente.

Dentro desses quatro modelos testados inicialmente, o que chamou a atenção foi a falta de predição de qualquer variável em impactar DAP induzida. Isto significa que nada impactou na disposição a pagar - induzidamente. Além desses modelos sugeridos pelo estudo, dois modelos de regressão alternativos foram apresentados (DAP Espontânea e Induzida), considerando-se todas as variáveis do modelo geral para cada DAP estudada.

A tabela 9 a seguir, apresentou os resultados das duas variáveis DAP's na equação do modelo geral. Os resultados apresentaram os seguintes valores de coeficiente de determinação: DAP_Esp; $R^{2}=$ 0,30 e DAP_Ind $R^{2}=0,05$. Na variável DAP Esp., as seguintes variáveis foram as preditoras: ÁREA, GINST, VLRUSO, VLREXIS, VLRHER, GOV e DESF, sendo a VLREXIS o maior impacto $B=, 38$. No modelo com a DAP induzida como dependente, AREA, RENP e PUSO foram preditoras, sendo RENP o maior impacto $B=, 241$. Nota-se que AREA foi a única variável significativa nos dois modelos de regressão, indicando ser importante nos modelos.

\begin{tabular}{c|c|c}
\hline VARIÁVEIS & \multicolumn{2}{|c}{ MODELO GERAL } \\
\hline & DAP ESPONTÂNEA & DAP INDUZIDA \\
\hline ÁREA &, $298^{* * *}$ &, $149^{*}$ \\
\hline IDADE &, 076 &,- 030 \\
\hline SEXO &,- 111 &, 020 \\
\hline GINST &, $132^{*}$ &,- 023 \\
\hline VINC &, 075 &, $241^{* * *}$ \\
\hline RENP &,- 035 &,- 019 \\
\hline RENF &, 111 &, 038 \\
\hline NPESS &,- 013 &
\end{tabular}

Continua... 


\begin{tabular}{c|c|c}
\hline DESF &, $132^{* *}$ &,- 068 \\
\hline FREQ &,- 006 &, 032 \\
\hline MOTIV &,- 012 &,- 008 \\
\hline EMANUT &,- 056 &,- 051 \\
\hline PERM &,- 059 &,- 062 \\
\hline PUSO &, 038 &, $115^{*}$ \\
\hline VLREXIS &, $381^{* * *}$ &,- 051 \\
\hline VLRHER &, $174^{* * *}$ &,- 032 \\
\hline VLRUSO &, $111^{*}$ &,- 011 \\
\hline VLROP &, 002 &, 041 \\
\hline DANAMB &, 000 &, 022 \\
\hline CMANUT &, 041 &,- 009 \\
\hline INTER &,- 027 &, 035 \\
\hline IMPOR &, 036 &, 064 \\
\hline GOV &, $102^{*}$ &, 050 \\
\hline OPESS &, 018 &,- 033 \\
\hline
\end{tabular}

Tabela 9 - Resultados dos Modelos Estimados das DAP's alternativas Nota: ${ }^{*} p<0,05 ;{ }^{* *} p<0,01 ;{ }^{* *} p<0,001$; valores Beta padronizados.

Fonte: Construção dos autores.

Este modelo geral incluiu diversas variáveis independentes. Isto se mostrou de uma riqueza grande no que tange a verificar ao máximo as variáveis preditoras da disposição a pagar. Assim, ÁREA foi a única a se apresentar significativamente nos dois modelos de DAP's. VLREXIS obteve valores positivos e alto de beta padronizado (em função de as pessoas serem individualistas, e atribuírem ao seu bem-estar condição única, o sinal esperado é positivo), GINST mostrou-se constante no modelo, apresentando resultados moderados, e GOV (o sinal esperado é positivo, porque perante a legislação o governo é o principal responsável pela preservação do meio ambiente em oferecer à sociedade uma qualidade de vida saudável) surgiu como importante na estrutura final.

Todavia, apesar das limitações inerentes aos modelos testados, sua utilização fornece subsídios importantes aos planejadores e tomadores de decisão interessados em obter informações quantitativas e qualitativas sobre os "bens e serviços" proporcionados pelo Horto Florestal de Rondonópolis - MT, em particular, na obtenção de recursos financeiros para a necessária preservação e conservação de seu estado natural.

Embora não tenham sido testados todos os potenciais "erros" 
presentes nesta pesquisa, a diminuição dos "erros" não é o único meio de garantir a validade da aplicação do MVC. De acordo com Mitchell e Carson, 1989; Pearce e Moran, 1994, citados por Obara e Santos (2000, p.121), "quatro tipos de testes têm sido recomendados para verificar a validade do Método".

Neste trabalho, levando-se em consideração todos os cuidados tomados na elaboração do questionário, e na verificação da validade do conteúdo, esta foi considerada satisfatória para a obtenção de DAP's confiáveis, visto que os entrevistados foram abastecidos com informações suficientes a respeito do Horto Florestal. Observou-se, através do comportamento deles, muita familiaridade com o Horto Florestal, haja vista que, por ocasião da pergunta "quanto ao nível de conhecimento que a população residente tinha sobre o bem em questão" todos alegaram conhecimento a respeito do Horto e apenas uma minoria (11,29\%), não o tinham visitado ainda.

Quanto à validade de critério, em se tratando de um Horto Florestal, sem a cobrança de ingresso na entrada, este critério não pode ser validado, por não existir um valor real para comparação com a DAP obtida.

O teste da validade por comparação, onde os resultados obtidos do MVC podem ser comparados aos valores obtidos por outros métodos, como os métodos "Custo de Viagem" e "Valor de Propriedade", ficou prejudicado pela inexistência de parâmetros suficientes, pelo fato de não ter sido realizado nenhum trabalho desta natureza no Horto Florestal.

Finalmente, a validade teórica tida pela fundamentação na observação dos resultados obtidos na pesquisa, com aqueles esperados na teoria, foi averiguada por meio da aplicação da técnica de análise de regressão.

\section{CONSIDERAÇÕES FINAIS}

O Horto Florestal de Rondonópolis, não poderia ser diferente das demais áreas de conservação quanto ao seu gerenciamento, pois a maior dificuldade é a carência de recursos financeiros. $E$, preocupado com o bem-estar da população que se utiliza do Horto Florestal, foi realizado este estudo de caso, procurando identificar 
nas diferentes características da população residente na zona urbana e rural de Rondonópolis, face à limitação da dotação orçamentária e na relevância de sua manutenção e preservação para as gerações atuais e futuras.

A análise dos resultados dos dados obtidos nesta pesquisa evidenciou a preocupação da população residente no município de Rondonópolis-MT em manter e conservar o Horto Florestal, ainda que tenha caráter eminente dos custos incorridos na oferta de bem-estar para a população em relação a um meio ambiente saudável e ao mesmo tempo servir de lazer para a sociedade em geral.

No presente estudo, com base na utilização do MVC considerando-se a equação (3), foi estimada uma $\mathrm{DAP}_{\text {total }}$ anual Espontânea e outra Induzida, obtidas pela multiplicação da disposição a pagar média (DAPM) de cada uma (espontânea e induzida) por uma estimativa da proporção da população da cidade relativa aos bairros existentes analisados na amostra, (MOTTA, 1998, p.27), tendo como população estimada pelo IBGE (2016) 218.899 habitantes, para uma população adulta (idade igual ou superior a 20 anos) de Rondonópolis-MT, em manter e preservar o Horto Florestal em seu estado natural.

Cálculo das DAP's: a) Cálculo da DAP Espontânea total anual levando-se em consideração a disposição a pagar média espontânea de R\$ 4,68 anuais, tem-se: DAPT Esp =4,68. (117/382). 218.899, e, DAPT Esp = R\$ 313.770,51. Este valor reflete a receita anual da disposição a pagar espontânea de apenas 30,71\% das pessoas entrevistadas, para a conservação e manutenção do Horto Florestal por um ano, através do recolhimento do IPTU; b) Cálculo da DAP Induzida total anual, considerando-se a disposição a pagar média induzida de $\mathrm{R} \$$ 9,85 anuais, tem-se: DAPT Ind= 9,85. (210/382). 218.899 e, DAPT Ind = R\$ 1.185.320,89.

A partir do momento em que um novo cenário foi apresentado aos entrevistados, ou seja, com melhorias na infraestrutura de lazer e recreação do Horto, incremento na programação cultural, educação ambiental, entre outras mais, as pessoas manifestaram interesse em pagar anualmente, na forma de imposto (IPTU), a receita acima, para contribuir na manutenção e conservação do Horto Florestal.

As limitações atribuídas à utilização do MVC estão relacionadas à vulnerabilidade do método a vários tipos de "erros" (erro hipoté- 
tico, de agregação, estratégico, de informação, de lance inicial, de método de pagamento, etc). No presente estudo, foi verificado as chances de diminuir ao máximo as possíveis fontes de "erros" nas diferentes etapas da pesquisa.

Vale esclarecer, também, que algumas questões foram observadas e merecem ser destacadas pelas limitações incorridas durante o período da pesquisa. Primeiro, observou-se que não há uma política efetiva de conservação e manutenção do Horto Florestal, pois em nenhum momento se cobra ingresso para acesso ao Horto e, diante das restrições orçamentárias públicas, sem o envolvimento da sociedade na disposição a pagar para mantê-lo e conservá-lo, torna-se inaceitável a gratuidade de utilização, pois a manutenção do Horto tem custos e também suas externalidades são atribuídas à sociedade.

Segundo, observou-se que muitas pessoas carecem de informações sobre o Horto Florestal e que estas não estão disponíveis. É necessária a elaboração de panfletos informativos sobre as funções dos recursos ambientais, bem como, apresentar aos usuários orientações sobre cuidados que devem ser tomados para preservação.

Finalmente, acredita-se que outros estudos, sendo realizados no Horto Florestal com maior profundidade e principalmente com outros métodos, em particular o Método de Preços Hedônicos, possam dar maior grau de confiabilidade aos dados apresentados nesta pesquisa, como subsídios à gestão pública, permitindo sobremaneira, decisões mais seguras, levando-se em consideração que este é o primeiro estudo na área de valoração realizado no Horto Florestal de Rondonópolis.

\section{REFERÊNCIAS}

ALENCAR FILHO, F. M. de; ABREU, L. M.; SILVA, T. B. M.; ROSSI, T. M. F.; ALENCAR, E. F. C. Valoração Contingente de Ativos Ambientais: Uma aplicação do método na área diretamente afetada pela Pequena Central Hidrelétrica PCH. KOTZIAN, em Júlio de Castilhos, RS. Revista Economia e Desenvolvimento, n. 25, vol. 1, 2013.

BARROS, M. I. A. de. Caracterização da visitação, dos visitantes e avaliação dos impactos ecológicos e recreativos do Planalto do Parque nacional do Itatiaia. Piracicaba - SP, 2003. 121 f. Dissertação (Mestrado em Recursos Florestais, com opção em Conservação de Ecossistemas Florestais) - Escola Superior de Agricultura "Luiz de Queiroz", Universidade de São Paulo. 
BISQUERRA, R., SARRIERA, J. C., MARTINEZ, F. Introdução à estatística: enfoque informático com o pacote SPSS. Porto Alegre: Artmed. 2004.

CARNEIRO, J. M. B.; MAGYAR, A. L.; GRANJA, S. I. B. Meio Ambiente, empresário e governo: conflitos ou parceria? Revista de Administração de Empresas, São Paulo: Abril, 1993.

CECHIN, A. e VEIGA, J. E. da. O Fundamento Central da Economia Ecológica. In: MAY, P. H. (Ed.). Economia do Meio Ambiente: Teoria e Prática. Rio de Janeiro: Elsevier. 2010.

COLE, D. N. Biophysical impacts of wildland recreation use. In: GARTNER, W. C.; LIME, D. W. (Ed.) Trends in outdoor recreation, leisure and tourism. New York: CABI Publishing, 2000. cap 23, p. 257-264.

CONSTANZA, Robert. Economia Ecológica: uma agenda de pesquisa. In. Valorando a natureza: análise econômica para o desenvolvimento sustentável. Peter Herman May e Ronaldo Serôa da Motta (Organizadores). Rio de Janeiro: Campus, 1994.

DHOLAKIA, UTPAL M. How effective are groupon promotions for businesses. Social Science Research Network, 2010.

HAIR, Joseph; ANDERSON, Rolphi; TATHAM, Ronald; BLACK, Willian. Multivariate Data Analysis. 5. Ed., Upper Saddle River: Prentice-Hall, 1998.

HAIR J. R., J. F.; BLACK, W. C.; BABIN, B, J.; ANDERSON, R. E.; TATHAM, R. L. Análise Multivariada de Dados. Porto Alegre: Bookman, Ed. 6, 688p. 2009.

HAIR, J. F., ANDERSON, R. E. TATHAM, R. L., BLACK, W. C. Análise Multivariada de Dados. Porto Alegre: Bookman, Ed.5, 593p. 2005.

HANLEY, N.; SPASH, C. L. Cost-Beneficit Analysis and the Environment. Hants, Inglaterra: Edward Elgar, 1993, 278 p.

HARDT, Letícia P. A. Paisagismo de praças e parques. In: Universidade Livre do Meio Ambiente. Curitiba: 1995.

IBGE - Instituto Brasileiro de Geografia e Estatística. Cidades (censo 2010). Disponível em: <htpp://www.ibge.gov.br/cidadesat/defaut.php>Acesso em: 21 fev. 2017.

JANSSON, J. Car(ing) for our environment?: Consumer eco-innovation adoption and curtailment behaviors: The case of the alternative fuel vehicle. Tese de Doutorado, Universidade de Umeå, Faculdade de Ciências Sociais, 123p. 2009.

MAIA, Alexandre Gori. Valoração de recursos ambientais. Campinas: $2002.182 \mathrm{f}$. Dissertação (Mestrado em Desenvolvimento Econômico, Espaço e Meio Ambiente) Área de Concentração: Economia do Meio Ambiente. Instituto de Economia. Universidade Estadual de Campinas.

MALHOTRA, Naresh K. Pesquisa de Marketing: Uma orientação aplicada. 6. ed. Porto Alegre: Bookman, 2011. 
MARCANTONIO, M. I. P.; SCHENKEL, S. Manejo Florestal - Uma Vantagem Competitiva: um estudo de caso de uma empresa produtora de tanino no sul do Brasil. In: SEMEAD - XIII Seminários em Administração, São Paulo, 13p. 2010.

MATO GROSSO, Secretaria de Estado do Meio Ambiente (SEMA), Base Cartográfica de MT, Cuiabá: SEMA, 2017.

MOTA, J. A.; BURSTZYN, M.; CÂNDIDO JUNIOR, J. O. e ORTIZ, R. O. A Valoração da Biodiversidade: Conceitos e Concepções Metodológicas. In: May, P. H. (Ed.). Economia do Meio Ambiente: Teoria e Prática. Rio de Janeiro: Elsevier. p.265-288.2010.

OBARA, A. T., SANTOS, J. E., BENZE, B. G. \& SCHUNK-SILVA, E. Valoração Contingente de Unidades de Conservação. Caso de estudo: Estação Ecológica de Jataí (Luiz Antônio, SP) in Estação Ecológica de Jataí. José Eduardo dos Santos e José Salatiel Rodrigues Pires (Editores). Vol. 1. São Carlos: RiMa, 2000.

PRADO, P.H.M. Avaliação do Relacionamento sob a ótica do cliente: Um estudo em bancos de varejo. Tese de doutorado: Fundação Getúlio Vargas, São Paulo, 2004.

ROMEIRO, A. R.; ANDRADE, D. C. Valoração Econômico Ecológico de Recursos Naturais. Revista Gestión y Ambiente. 12 (3): p.21- 36. 2009.

SANCHES, Keila Lima. Uma abordagem econômica ao marketing verde no Bioma Cerrado. 2014. xvii, 140 f., il. Tese (Doutorado em Engenharia Florestal). Universidade de Brasília, Brasília, 2014.

TABACHNICK, B. G.; FIDELL, L. S. Experimental designs using ANOVA. Thomson/ Brooks/Cole. 2007. 